\title{
Au huitième jour, la verve de Welles s'empara du monde
}

\section{Guillaume Bourgois}

\section{OpenEdition}

12 Journals

Édition électronique

URL : https://journals.openedition.org/recherchestravaux/723

DOI : 10.4000/recherchestravaux.723

ISSN : 1969-6434

Éditeur

UGA Éditions/Université Grenoble Alpes

\section{Édition imprimée}

Date de publication : 15 décembre 2014

ISBN : 978-2-84310-291-2

ISSN : 0151-1874

\section{Référence électronique}

Guillaume Bourgois, «Au huitième jour, la verve de Welles s'empara du monde », Recherches \& Travaux [En ligne], 85 | 2014, mis en ligne le 15 décembre 2014, consulté le 29 octobre 2021. URL : http:// journals.openedition.org/recherchestravaux/723; DOI : https://doi.org/10.4000/recherchestravaux. 723

(c) Recherches \& Travaux 
Guillaume BourgoIs

Univ. Grenoble Alpes

\section{Au huitième jour, la verve de Welles s'empara du monde}

FALSTAFF : Give me a cup of sack to make my eyes look red, that I may be thought I have wept; for I must speak in passion [...].

William SHAKESPEARE ${ }^{1}$

Dès ses débuts, Orson Welles démontre un intérêt particulier pour la verve, en tant qu'élément permettant d'augmenter la force persuasive du discours. Le 30 octobre 1938 , sur les ondes de la radio CBS, le futur cinéaste organise l'une des plus célèbres supercheries de l'histoire médiatique américaine. Il lit et fait lire à ses collaborateurs du Mercury Theatre (la troupe de théâtre qu'il dirigea pendant ses premières années d'activité et qui l'accompagnera dans toute sa carrière) des extraits du roman de science-fiction d'H. G. Wells La Guerre des mondes, de façon si convaincante qu'une grande partie des auditeurs crut réellement que des extraterrestres venus de Mars étaient en train d'envahir les États-Unis - on raconte même qu'une jeune femme se rendit en haillons dans un commissariat et prétendit avoir été attaquée par des petits hommes verts. Le succès de la supercherie était dû à la forme à travers laquelle était transmis le discours des comédiens - les extraits de La Guerre des mondes étaient lus par flashs info successifs, qui venaient interrompre un concert de musique classique - mais surtout à la verve de Welles et de ses collaborateurs, dont le souffle et le rythme expressif surent convaincre les auditeurs et transformer la matière fictionnelle en preuve d'une invasion.

Cependant, la collaboration de Welles à un projet mené par son ami Ernest Hemingway un an auparavant révèle, à côté d'une réelle fascination pour la

I. W. Shakespeare, Henry IV, partie I, acte II, scène IV 
faculté de la verve à faire bouger les frontières entre fiction et réalité, une certaine méfiance envers cette même faculté lorsqu'elle est utilisée à des fins politiques. En 1937, plusieurs intellectuels américains de gauche, parmi lesquels Hemingway et Dos Passos, entreprirent de réaliser, sur la Guerre d'Espagne qui faisait rage à l'époque, un documentaire intitulé Terre d'Espagne, dans le but de convaincre l'opinion publique américaine de la nécessité d'une intervention aux côtés de l'armée républicaine. Hemingway et Dos Passos rédigèrent pour l'occasion un commentaire destiné à être entendu off sur des images tournées en Espagne par le cinéaste Joris Ivens. L'auteur de Pour qui sonne le glas demanda à Welles d'assurer la voix off et ce dernier lut le texte avec une neutralité qui ne convainquit absolument pas les intellectuels à l'origine du projet - dans la version définitive de Terre d'Espagne, ce sont Hemingway et Dos Passos eux-mêmes qui lisent le commentaire sur un ton enflammé. Le choix d'une diction blanche de la part du futur cinéaste obéit à deux objectifs. D'une part, il s'agissait de souligner le réalisme factuel des images tournées par Ivens. D'autre part, bien que très proche des milieux de gauche et convaincu qu'il fallait envoyer des troupes américaines en Espagne, Orson Welles entendait montrer que la verve constitue un puissant outil de manipulation.

La filmographie wellesienne est construite sur l'ambivalence de la verve que révèlent ces deux exemples - elle est à la fois un moteur esthétique et un élément dangereux.

En I94I sort le premier long-métrage de Welles, Citizen Kane, unanimement considéré comme l'un des plus grands chefs-d'œuvre de l'histoire du cinéma. Avec ce portrait caché du magnat de la presse William Randolph Hearst, le cinéaste compose un film dans lequel la parole fonctionne comme un catalyseur esthétique - ce qui fait dire à Youssef Ishaghpour, auteur d'une somme consacrée à l'œuvre de Welles, que Citizen Kane représente le premier grand film de l'ère du parlant, de la même manière que Naissance d'une nation (1915) de Griffith fut le premier grand film du cinéma muet ${ }^{2}$.

Homme de discours enflammés, aussi bien prononcés lors de meetings politiques qu'au détour d'une simple conversation entre amis, le «citoyen Kane» interprété par Welles entraîne ses proches et ses collaborateurs dans un tourbillon verbal contaminant l'image et le son - comme le prouve la longue séquence centrale montrant la prise en main progressive du journal L'Inquirer par le personnage principal. Au fur et à mesure que Kane développe avec passion sa vision du futur de L'Inquirer et réduit l'ancien rédacteur en chef au

2. Y. Ishaghpour, Orson Welles cinéaste, une caméra visible I: mais notre dépendance à l'image est énorme, Paris, Éditions de la Différence, 200I, quatrième de couverture. 
silence, les contre-plongées interviennent de façon massive, le montage devient plus fragmenté, l'image se surcharge d'éléments hétéroclites apportés par les serviteurs du personnage principal (un lit, des tableaux, etc.), et un brouhaha sonore est produit par les allées et venues de ces derniers. Plus la scène avance et moins la séquence est objective. Elle finit, non par donner à voir l'espace de la salle de rédaction contaminé par la parole vive de Kane, qui perturbe les repères spatiaux, sonores, et se produit la rencontre visuelle entre les effets intimes du personnage et le lieu publique qui apparaît à l'écran, tout comme la verve peut constituer le moyen de colorer un discours public d'une marque personnelle. Globalement, le verbe du personnage fonctionne également comme un catalyseur temporel. L'arrivée du personnage à la tête de L'Inquirer produit un bon dans le tirage du journal, et ses gestes et décisions n'ont de cesse d'accélérer les déplacements à l'image de ses collaborateurs et de dynamiser le déroulement temporel du film, ce que traduit l'utilisation de plusieurs fondus enchaînés marquant des ellipses dans le temps, comme pour signifier que l'action physique et verbale du "citoyen Kane» est source d'une accélération telle qu'il est impossible de la suivre en direct. En vertu d'une de ces ellipses temporelles, le passage se déroulant dans la salle de rédaction est en deux temps, qui correspondent à deux moments de la vie de L'Inquirer : une première scène montre l'arrivée de Kane à la tête du journal; une seconde un banquet organisé quelques années plus tard lorsque le journal devient le plus lu des États-Unis. L'usage massif dans la seconde des effets cinématographiques progressivement mis en place dans la première (contre-plongée, montage fragmenté, surcharge visuelle et sonore) témoigne de la contamination définitive de la salle de rédaction, et du journal dans son ensemble, par le verbe passionné du «citoyen Kane».

C'est précisément à ce moment que Citizen Kane fait apparaître le péril que constitue la verve du personnage central. Plusieurs plans font apparaitre une discussion entre les deux amis et collaborateurs principaux de celui qu'interprète Welles : Leland (Joseph Cotten) et Bernstein (Everett Sloane). Initialement, on aperçoit Kane qui danse au second plan; puis, au gré d'un changement d'axe, ce n'est plus Kane qui apparaît directement au fond de l'image mais uniquement son reflet dans une vitre. Par ce jeu avec l'arrière-plan, le personnage principal est caractérisé comme quelqu'un pour qui il n'y a pas de différence entre un élément et son image, entre la chose vraie et sa reproduction. Dans l'une des scènes précédentes, Kane décidait de publier une "pétition de principe» en une de L'Inquirer. Leland lui demandait s'il pouvait garder le manuscrit du texte. En réclamant l'original, Leland signifie implicitement qu'il ne suffit pas de publier un texte pour que celui-ci soit vrai. Il prend ainsi ses distances avec le personnage principal et oblige à considérer Kane comme un homme du simulacre, dont la verve, par sa capacité à faire bouger les frontières entre 
réalité et fiction, est l'arme de prédilection. Parce qu'il a remodelé L'Inquirer à l'image de son verbe, le personnage principal a créé un organisme monstrueux : il est dit pendant le banquet que le journal a relayé des mensonges qui ont précipité la guerre entre les États-Unis et l'Espagne - ce qui renvoie à la responsabilité de l'organe de presse dirigé par William Randolph Heart dans le conflit américano-hispanique de I898 - et les rédacteurs du journal, qui s'amusent à utiliser des fusils en bois comme des instruments de musique, se comportent comme de grands enfants qui sacrifient toute considération morale ou de déontologie journalistique à la force expressive et manipulatrice de la verve de Kane.

La célèbre séquence initiale révèle que c'est avant tout le spectateur qui est prisonnier de la parole du personnage principal. Par une utilisation massive de gros plans sur la main et la bouche de Kane, le passage donne au personnage central des dimensions monstrueuses et en fait une figure de dieu moderne. Il tient dans sa main une boule à neige, qu'il laisse tomber au moment de sa mort. L'objet se brise et l'on voit l'arrivée de l'infirmière venue s'occuper de Kane. L'image de l'entrée de la jeune femme est montrée à travers la perspective déformante d'un morceau de verre de la boule à neige au sol. Ceci annonce au spectateur qu'il ne pourra jamais accéder à une image objective du personnage. Au contraire, il est condamné à voir l'existence de celui qu'interprète Welles sous l'influence de la verve déformante et manipulatrice de Kane.

Le personnage principal expire en prononçant le mot «Rosebud», sur lequel les deux journalistes au cour du film tentent de faire la lumière afin de mieux comprendre le "citoyen Kane». Leur enquête échoue et ils n'identifient pas la signification ni l'origine du mot. Dans la dernière séquence, l'un des journalistes conclut qu' «un mot ne permet pas d'expliquer la vie d'un homme». De ce point de vue, il faut comprendre que dans Citizen Kane, ce n'est pas le contenu des discours incessants du personnage principal qui importent, mais avant tout la force et le style expressifs qui les portent, car c'est grâce à eux et à leur impact sur celui qui écoute que le «citoyen Kane» fait bouger la frontière entre vérité et mensonge.

Figure archétypale dans la filmographie de Welles, Kane est le premier d'une longue série de personnages interprétés par le cinéaste et caractérisés par un verbe tonitruant - à commencer par le mystérieux Mr. Arkadin de Dossier secret (1955). Alter ego de Kane, le richissime trafiquant d'armes qu'est Arkadin s'entoure d'une cour qu'il charme à longueur d'anecdotes et de traits d'esprit. Le Littré indique que la verve est associée à l'idée de caprice et Dossier secret le rappelle à deux niveaux. 
Arkadin est un être capricieux, qui décide de la vie et de la mort de ses anciens collaborateurs sur un coup de tête. De plus, il organise un bal costumé dans son château espagnol. L'un des invités annonce au personnage principal que les masques des participants sont inspirés des figures inquiétantes des œuvres de Goya. Le passage se place ainsi sous l'égide des Caprices, série de gravures du peintre espagnol, représentant des monstres terrifiants. Le style de Welles, qui exacerbe les contrastes afin de créer un clair-obscur très marqué, ainsi que les mouvements et axes de caméra qui viennent renforcer l'aspect grotesque des masques des invités, se veut au plus près de l'esthétique des gravures. Plusieurs masques sont clairement inspirés de certains Caprices, comme la tête d'âne qu'arbore l'un des convives, qui rappelle le Caprice $\mathrm{n}^{\circ} 39$ - image d'un homme à tête d'âne, sous laquelle figure l'inscription "ainsi était son grand-père», façon pour Goya de dénoncer l'idiotie des hidalgos désargentés, qui s'obsèdent à mettre en avant la noblesse de leur lignée. Le bal costumé aux accents de Caprices permet de comprendre l'image d'une chauve-souris géante en carton-pâte, qui apparaît à la fin du générique de Dossier secret. L'animal est présent sur le Caprice ${ }^{\circ} 43$ de Goya, surtout connu pour l'inscription qui l'accompagne, "El sueño de la razon produsce monstros» ("Le sommeil de la raison produit des monstres»). Si le verbe de Kane colorait progressivement l'espace de la salle de rédaction de L'Inquirer, Arkadin enferme ses proches ainsi que le spectateur dans un lieu où la réalité est déformée, vue à travers le prisme des Caprices goyesques. Les Caprices fonctionnent comme un voile placé sur le monde, qui relaie la noirceur de la vision du monde au cœur des discours successifs du personnage central.

Dossier secret révèle en outre que l'utilisation de la verve chez Welles s'inscrit dans une lutte contre le néant. À la fin du film, lorsque s'effondrent les remparts narratifs, visuels et discursifs mis en place par Arkadin afin d'empêcher les autres personnages de découvrir la vérité à son sujet, et donc de sortir de l'emprise de son verbe, le trafiquant d'armes ne meurt pas, il disparait mystérieusement. La défaite de la verve du personnage équivaut à une disparition dans le néant. C'était déjà le sens de l'une séquence de l'adaptation de Macbeth par Welles en 1947. Au moment où l'emprise verbale du personnage central, interprété par le cinéaste, s'affaiblit, il prend conscience de la vanité de l'existence et prononce la célèbre tirade : "Lifés but a walking shadow, a poor player that struts and frets his hour upon the stage. And then is heard no more. It is a tale tod by an idiot, full of sound and fury signifying nothing." " La citation de Shakespeare est entendue off, alors qu'une scène vide apparaît à l'écran, symbolisant le

3. «La vie n'est qu'une ombre en marche, un pauvre acteur, qui se pavane et se démène son heure durant sur une scène, et puis qu'on n'entend plus. C'est un récit conté par un idiot, plein 
néant dans lequel s'apprête à sombrer Macbeth. Le personnage wellesien est un personnage maudit, qui existe et s'affirme par l'utilisation de la verve, mais est à terme condamné à être happé par le néant.

En parallèle, Citizen Kane laisse entendre que la verve est avant tout une question corporelle. Après la mort du personnage principal au début du film, sont montrées des images d'archives retraçant sa vie. Les images sont muettes; la première fois que Kane est à l'image, il gesticule avec énergie lors de l'un de ses discours. Le passage suggère que c'est surtout en utilisant son corps pour véhiculer les mots qu'il employait qu'il a pu influencer et manipuler son entourage. La façon dont la verve déborde la parole et concerne le corps est clairement posée par certains dictionnaires. Le TLF, par exemple, mentionne un passage d'Hugo : "Alors on se pâma; Mars embrassa Minerve, Mercure prit la taille à Bellonne avec verve.» (Hugo, Légende, t. II, I859, p. 857.)

Au fil des œuvres de Welles, le spectateur suit l'évolution d'un corps, celui du cinéaste qui gagne de plus en plus de poids. Loin d'être anecdotique, la transformation de la silhouette de Welles pose un lien évident entre la parole et le corps. La verve est un principe de déséquilibre entre la teneur objective d'un discours et son impact et le cinéaste s'appuie sur son propre corps pour créer des déséquilibres visuels accompagnant la parole de ses personnages. La séquence finale de La Soif du mal (1958) en témoigne. Elle montre le règlement de compte entre les deux personnages principaux : le vertueux Vargas, interprété par Charlton Heston, et le corrompu Quinlan, campé par Welles. Le premier cherche à obtenir une confession du second, qu'il a placé sur écoute. Au lieu d'offrir à Vargas cette preuve irréfutable, c'est-à-dire une déclaration objective au sein de laquelle il n'y aurait aucun écart entre les mots et leur signification, Quinlan se lance dans un discours enflammé et cherche à perdre et Vargas et le spectateur dans ses volutes verbales. Au niveau sonore, un écho est créé entre les mots prononcés par le premier puis ceux entendus à travers le poste radio du second, qui inscrivent cet écart contre lequel Vargas voulait lutter. La séquence recrée au niveau visuel le déséquilibre que produit la parole de Quinlan, en utilisant massivement des axes en plongée et contre-plongée, des axes de biais, et en fragmentant le découpage. À plusieurs reprises, la caméra passe brutalement d'une contre-plongée à une plongée ou vice-versa, comme le plateau d'une balance sur laquelle on ajouterait ou on enlèverait un poids. Ceci fait des déséquilibres visuels de la séquence des conséquences de la différence de poids entre la maigre Vargas et l'obèse Quinlan.

de bruit et de fureur, Et qui ne signifie rien.» (W. Shakespeare, Macbeth, acte V, scène v, dans Tragédies II, trad. J.-M. Déprats, Paris, Gallimard, coll. «Bibliothèque de la Pléiade», p. 485. 
La différence de poids est également au cœur d'un travail wellesien sur des éléments venus de la tauromachie - il faut rappeler que le cinéaste était un grand aficionado. Au début de la scène, un miroir reflète l'image de Vargas et la glace se trouve au milieu de portraits de toreros, associant celui qu'interprète Charlton Heston à un matador. Des cornes de taureau sont accrochées au-dessus de plusieurs portes dans la chambre qu'occupe Quinlan, ce qui l'assimile à un toro de combat. Le rapprochement est renforcé par le fait que le personnage se plaint de ne pas bien voir, caractéristique des animaux utilisés pour les corridas. L'affrontement entre Vargas et Quinlan qui clôt le film s'achève de façon traditionnelle par la mort du toro joué par Welles, mais son déroulement est loin d'être canonique. Alors que dans une corrida, le torero leurre le toro au gré de ses passes de muleta, c'est ici le matador qui est leurré par la vivacité du discours de son adversaire. Avec cette scène finale de La Soif du mal, Welles démontre que pour lui la verve provient d'une part animale et intuitive du corps humain, par laquelle celui qui l'emploie déséquilibre la perception auditive et visuelle de ceux qui l'entourent.

Quinlan est un personnage extrêmement ambigu. Policier corrompu obtenant des confessions sous la menace et forgeant de fausses preuves, on découvre à la fin du film qu'il était le seul à avoir raison concernant l'affaire sur laquelle il enquêtait aux côtés de Vargas. La Soif du mal signifie implicitement que la verve peut avoir une utilité, ici celle de s'opposer au rationalisme trop strict de Vargas, mais elle ne doit pas être utilisée seule. Dans le prolongement de cette idée, Falstaff, sorti sept ans pus tard, met en place une véritable dialectique de la verve.

Le récit de Falstaffs' inspire de plusieurs pièces de Shakespeare faisant intervenir le personnage-titre : Richard II, Henry IV, Henry V et Les Joyeuses Commères de Windsor. Welles y interprète le truculent Falstaff, coureur de jupons et buveur invétéré, qui mène dans ses aventures rocambolesques le prince Henry, fils du roi Henry IV et héritier de la couronne. Deux séquences sont construites en miroir : la première montre le roi au palais donnant des ordres à ses serviteurs, et la seconde Falstaff qui s'amuse à imiter le souverain dans une taverne, une casserole sur la tête en guise de couronne. Certains plans obéissent exactement à la même construction géométrique, mais à nouveau la silhouette de Welles, monstrueuse en comparaison à celle du filiforme comédien interprétant Henry IV, déséquilibre la composition. Par ses mots d'esprit et sa gestuelle, Falstaff ridiculise le discours politique et la mise en scène qui l'entoure. Sa verve est une force anarchique qui détruit tout sur son passage, et met en lumière l'horreur des décisions prises par le souverain, notamment celle de plonger le pays dans la guerre civile afin de défendre ses propres intérêts. Devenu malgré lui recruteur pour l'armée, le personnage principal ne choisit des soldats qu'au 
gré de jeux de mots sans aucun lien avec les capacités physiques des candidats retenus. Quelques minutes plus tard, la principale scène de bataille est montrée en accéléré, comme si elle était contaminée par la vivacité et l'ironie du verbe de Falstaff - ce qui empêche toute tonalité épique et dénonce l'absurdité de la campagne militaire. Flaubert écrivait dans une lettre à Louise Collet : "Quand est-ce qu'on écrira les faits au point de vue d'une blague supérieure, c'est-à-dire comme le bon Dieu les voit, d'en haut ${ }^{4}$ ?" À travers le personnage de Falstaff, particulier dans l'œuvre de Welles car n'utilisant pas la verve pour asseoir sa domination sur son entourage, le spectateur perçoit le monde au point de vue d'une blague supérieure.

Le film de 1965 est en même temps un récit initiatique, celui du prince Henry, qui commence par accompagner Falstaff dans sa vie de plaisirs, au grand dam du roi, puis finit par accepter la couronne et ses responsabilités à la mort de son père. La voix off finale annonce au spectateur que le roi Henry V fut un roi d'une grande sagesse. Cette sagesse résulte à la fois des enseignements de Falstaff, dont la verve a montré au prince ce que la pratique du pouvoir de son père avait de ridicule et d'injuste, et d'une mise à distance de l'ironie de celui qu'interprète Welles. En effet, dans l'une des scènes les plus bouleversantes de la filmographie du cinéaste, le prince devenu roi répudie son ancien ami - répudiation symbolisée par l'utilisation d'un champ-contrechamp qui interdit à Falsaff de s'approcher du souverain. En conséquence du geste d'Henry V, le personnage central meurt quelques minutes plus tard. Son corps, enfermé dans un cercueil noir aux lignes géométriques strictes, est désormais invisible et ne peut déséquilibrer la composition du cadre, signe que l'ironie de Falstaff s'est tue à jamais.

Dernier film majeur de la filmographie wellesienne, F for fake (1973) - Vérités et mensonges en français - offre une ultime variation autour de la question de la verve. L'œuvre est un vrai faux reportage consacré à l'un des plus célèbres faussaires de l'histoire de la peinture, le Hongrois Elmyr de Hory, ainsi qu'à son biographe non-officiel Clifford Irving. Les deux hommes s'expriment face caméra et $F$ for fake organise la lutte entre les propos enflammés d'Elmyr de Hory et Clifford Irving, qui se contredisent et tentent chacun de manipuler le spectateur. Dans le cas du faussaire, la verve est également un principe de création picturale. De la même façon qu'il parle avec brio pour faire accepter des mensonges, il peint à la manière virtuose de Picasso ou Van Dongen dans le but de tromper les experts des plus grands musées européens et américains.

4. G. Flaubert, Lettre à Louise Collet du 7 octobre I852, Correspondance, t. II, Paris, Gallimard, coll. «Bibliothèque de la Pléiade», p. I68. 
Welles laisse se déployer la verve verbale et picturale d'Elmyr de Hory, tout en procédant à d'incessants arrêts sur images, qui la tiennent en échec et la dénoncent. En parallèle, le cinéaste joue dans le film et semble dédoublé : dans certaines séquences, il évoque sa vie avec passion, mêlant subtilement faits avérés et légendes qui ont circulé à son sujet; dans d'autres, il dénonce ses mensonges. L'un des premiers auteurs modernes du cinéma américain, aux côtés d'Hitchcock, Orson Welles place ainsi $F$ for fake et l'ensemble de sa filmographie au croisement entre le cinéma hollywoodien, où règnent la manipulation et l'écart entre réalité historique et légendes, et le cinéma européen, en particulier celui de la Nouvelle Vague, qui ne cesse de déconstruire les agencements des œuvres filmiques afin de s'opposer aux manipulations.

À travers ses personnages hâbleurs et la singularité de son style visuel, Welles a fait de la verve, à la fois fascinante et dangereuse, l'élément central d'une révolution au sein de l'esthétique classique, qui permettra quelques années plus tard l'arrivée de la génération du Nouvel Hollywood. Francis Ford Coppola, l'un des plus grands représentants de cette génération, se souviendra de l'importance du réalisateur de Citizen Kane dans son film Apocalypse Now (1979). Non seulement l'œuvre de 1979 est une adaptation du texte de Conrad Au coeur des ténèbres, que Welles a longtemps envisagé de transposer à l'écran, mais le personnage de Kurtz (Marlon Brando), dont les discours scandent le film et dont le poids ne cesse de déséquilibrer la composition et l'axe des plans, est hanté par les spectres du mystérieux Kane, de l'inquiétant Arkadin, du monstrueux Quinlan et de l'anarchisant Falstaff.

5. Il suffit de penser à la phrase de L'Homme qui tua Liberty Valance (1962) de John Ford: «When legend becomes fact, print the legend» - traduite dans la version française par «Si la légende est plus belle que la réalité, imprimez la légende». 